\title{
Increased primary autoimmune thyroid diseases and thyroid antibodies in sarcoidosis: evidence for an under-recognised extrathoracic involvement in sarcoidosis?
}

\author{
Foteini Malli, ${ }^{1}$ Alexandra Bargiota, ${ }^{2}$ Kathrin Theodoridou, ${ }^{3}$ Zoi Florou, ${ }^{3}$ Fotini Bardaka, ${ }^{1}$ \\ George N. Koukoulis, ${ }^{2}$ Konstantinos I. Gourgoulianis, ${ }^{1}$ Zoe Daniil ${ }^{1}$
}

${ }^{1}$ Respiratory Medicine Department, ${ }^{2}$ Clinic of Endocrinology and Metabolic Diseases, ${ }^{3}$ Department of Immunology and Histocompatibility, University of Thessaly, Medical School, Larissa, Greece

\begin{abstract}
OBJECTIVE AND DESIGN: Sarcoidosis has been associated with thyroid diseases. However, until today no definite conclusions have been drawn. We aimed to assess the frequency of thyroid disorders and the levels of thyroid hormones and thyroid antibodies in 68 sarcoidosis patients and 75 controls. Additionally, we performed ultrasonography and fine-needle aspiration. RESULTS: In this prospective case control study conducted in the University Hospital of Larissa, Greece, overt thyroid disease was present in $29.4 \%$ of patients and $16.1 \%$ of patients presented clinical autoimmune thyroid disease. Sarcoidosis patients had a significantly higher frequency of serological autoimmunity. Female patients had significantly increased frequency of positive TSH receptor antibodies (TRAbs) and antithyroid peroxidase antibodies (TPOAbs) when compared to gender-matched controls $(40 \%$ vs $0 \%, p<0.001$, and $28.8 \%$ vs $11.86 \%, p=0.029$, respectively). The hypoechoic pattern of the thyroid was more frequent in female patients vs controls $(p<0.001)$. Male patients had a higher frequency of TRAbs and hypoechoic pattern of the thyroid gland $(43.4 \%$ vs $0 \%, p=0.002$, and $39.1 \%$ vs $6.25 \%, p=0.021$, respectively). Indices of thyroid autoimmune disease were significantly more frequent in sarcoidosis patients vs gender-matched controls. Increased TPOAbs were significantly associated with clinical autoimmune disease in sarcoidosis. CONCLUSIONS: Overall, the findings derived from this study suggest that thyroid disorders are frequent in sarcoidosis. This association may potentially be the result of increased thyroid antibodies.
\end{abstract}

Key words: Autoimmunity, Sarcoidosis, Thyroid, Thyroid antibodies 


\section{INTRODUCTION}

Sarcoidosis is a chronic systemic disease affecting, in the majority of cases, the respiratory system that is characterized histologically by the presence of non-caseating granulomas. ${ }^{1}$ Its pathogenesis remains elusive, but existing data suggest an association of sarcoidosis with an exaggerated cellular response to antigens. ${ }^{2}$ Data indicate that patients with sarcoidosis exhibit an enhanced immune response resulting from increased $\mathrm{T}$ cell activity, while the disease is associated with increased humoral response and hyperglobulinemia. ${ }^{3}$

Sarcoidosis has a diverse range of clinical presentations including not only respiratory but also opthalmological and dermatological manifestations. Vitamin D and calcium metabolism disorders are also common findings in patients with sarcoidosis. ${ }^{4}$ Moreover, several studies have demonstrated a high prevalence of different autoantibodies (antinuclear antibodies to double-stranded DNA and rheumatoid factor) in sarcoidosis patients and the co-existence of sarcoidosis with autoimmune diseases. ${ }^{5-7}$

An association between sarcoidosis and thyroid disorders has also recently been reported in the literature. Although granuloma infiltration of the thyroid gland is relatively uncommon, an increased prevalence of clinical and subclinical hypothyroidism and Graves' disease has been described in patients with sarcoidosis. ${ }^{8}$ However, the results remain conflicting, with most of the published data limited to case reports, ${ }^{9}$ and in only one study has a complete thyroid work-up been performed. ${ }^{8}$ Controversy also exists regarding the presence of thyroid antibodies in sarcoidosis patients, with studies pointing to increased prevalence of antithyroid peroxidase antibodies (TPOAbs) and antithyroglobulin antibodies $(\mathrm{TGAbs})^{10}$ in sarcoidosis patients, while others have failed to document any differences. ${ }^{11}$ Data concerning TSH receptor antibodies (TRAbs) are rather scarce, most of them derived from case reports; meanwhile, in a prospective study by Antonelli et $\mathrm{al}^{12}$ the authors failed to demonstrate increased TRAbs titles in sarcoidosis. Thus, currently no definite conclusions can be drawn regarding the association of sarcoidosis with thyroid disorders. The aforementioned findings indicate an increased prevalence of thyroid disorders in sarcoidosis but highlight the necessity for further studies in order to elucidate the prevalence of thyroid disease in sarcoidosis patients and the nature of their co-existence. Therefore, the aim of the present study was to determine the frequency and the type of thyroid disorders in a group of patients with sarcoidosis.

\section{METHODS}

\section{Subjects}

The present prospective case control study was conducted in the University Hospital of Larissa, Greece. Patients were recruited by consecutive sampling from the Respiratory Outpatient Clinic of the University Hospital of Larissa, Greece, from November 2007 to December 2010. A control group of healthy volunteers, matched in terms of age with patients, was also recruited over the same period. The diagnosis of sarcoidosis was based on standard published criteria. ${ }^{1}$ All patients had biopsy-proven sarcoidosis with histopathologic confirmation of non-caseating granulomas. None of the patients was under corticosteroid and/ or other immunosuppressive therapy at the time of the study. Subjects with other autoimmune diseases were excluded from the study. In order to exclude differences in iodine intake, only subjects residing in our geographical area for more than two decades were recruited in both groups, as studies conducted in the area have indicated a sufficient iodine intake for the last 20 years. ${ }^{13,14}$

A detailed medical history was obtained from all subjects with emphasis on risk factors for thyroid disorders (family history of thyroid disease, radiation exposure and previous residence in iodine-deficient areas). For sarcoidosis patients, symptoms (MRC dyspnea scale, cough), clinical examination and radiological findings were recorded. Arterial blood gases (model 1630; Instrumentation Laboratories, Milan Italy), pulmonary function tests (Bodyplethysmograph, Master-Screen Body, Viasys Healthcare, Germany), SACE, serum calcium and $24 \mathrm{~h}$ urine calcium measurements were also carried out. Sarcoidosis patients with positive thyroid antibodies and a previous diagnosis of thyroid autoimmune disorder (i.e. Hashimoto's thyroiditis, Graves' disease) who were under proper treatment when this study was performed were classified as suffering from clinical autoimmune thyroid disease (AITD). 
Ethics committee approval and written informed consent from all subjects were obtained.

\section{Laboratory measurements}

Venous blood was drawn from all participants in order to assess thyroid function and antibodies. Thyroid stimulating hormone (TSH), triodothyronine (T3) and free thyroxine (FT4) were determined using an electrochemiluminescent immunoassay analyzer (Hitachi E170, Roche Diagnostics Gmbh, Mannheim, Germany). Controls from Bio-Rad were used for T3, fT4, and TSH protocols (Bio-Rad Laboratories, Irvine, CA, USA). Intraassay coefficients of variation were 4.8, 4.9, and 3.7\% for T3, fT4, and TSH, respectively. Appropriate enzyme-linked immunosorbent assays (ELISAs) were used for the measurement of TPOAbs (QUANTA Lite TPO, INOVA Diagnostics Inc., San Diego, USA), TGAbs (QUANTA Lite Thyroid T, INOVA Diagnostics Inc., San Diego, USA) and TRAbs (Medizym TRAb clone, Medipan, Berlin, Germany) in serum with intraassay coefficients of variation $8.5,7.4$ and $16 \%$, respectively. TRAbs, TPOAbs and TgAbs positivity was set up at $1 \mathrm{IU} / 1,50$ $\mathrm{U} / \mathrm{ml}$ and $200 \mathrm{U} / \mathrm{ml}$, respectively. To examine whether the prevalence of thyroid antibodies correlated with disease activity, we assessed the circulating levels of serum ACE.

\section{Ultrasonography}

A thyroid ultrasound (GE Medical Systems-Vivid 3, Milwaukee, WI, USA) with a 7.5 MHz transducer was performed in all participants by a single physician (A.B.) who was blinded to the subject's disease status and laboratory measurements. Thyroid volume was calculated using the volumetric ellipsoid method (height $\mathrm{x}$ width $\mathrm{x}$ depth $\mathrm{x}$ correction factor 0.479 ). ${ }^{15}$ Structural abnormalities in the thyroid gland associated with thyroid autoimmunity were scored as previously reported. ${ }^{16}$ The presence of hypoechoic and dyshomogeneous echogenecity was rated arbitrarily at three levels $(0=$ normal echogenicity; $1=$ slight hypoechoic and dyshomogeneous; $2=$ severely hypoechoic and dyshomogeneous). The presence of thyroid nodules was recorded and nodules with a diameter greater than $10 \mathrm{~mm}$ were subjected to fineneedle aspiration (FNA) for cytological analysis. All FNAs were performed by the same physician (A.B.).

\section{Statistical analysis}

Data are presented as mean \pm SD unless otherwise indicated. Normal distribution was assessed by the Kolmogorov-Smirnov test. Comparison between patients and controls was performed with the use of Student's t test or Mann-Whitney U-test according to variable distribution. The $\mathrm{x} 2$ test was used to compare categorical variables. Univariate correlations were performed by Pearson's correlation coefficient for variables that were normally distributed or by Spearman's correlation coefficient for variables that were not normally distributed. A p value of $<0.05$ was considered to be statistically significant. Analysis was performed using the SPSS 16 statistical package (SPSS Chicago, IL).

\section{RESULTS}

The study population consisted of 68 sarcoidosis patients (mean age: 53.08 \pm 11.6 years, 23 of them males) and 75 healthy volunteers (mean age: $50.78 \pm 13.57$ years, 16 of them males). Anthropometric characteristics and baseline measurements of participants are shown in Table 1. Thyroid disease was present in $29.4 \%$ of patients: 10 had autoimmune (Hashimoto's) thyroiditis with hypothyroidism, 4 had multinodular goiter, 4 had solitary thyroid nodule, 1 had Graves' disease and 1 had papillary follicular thyroid cancer. Overall, $16.1 \%$ of the patients had clinical AITD, as was indicated by positive titers of thyroid antibodies

Table 1. Anthropometric characteristics and baseline measurements of study participants

\begin{tabular}{lcc}
\hline Parameter & $\begin{array}{c}\text { Controls } \\
(\mathbf{n = 7 5})\end{array}$ & $\begin{array}{c}\text { Sarcoidosis } \\
\text { patients } \\
(\mathbf{n = 6 8})\end{array}$ \\
\hline Age (years) & $50.78 \pm 13.57$ & $53.08 \pm 11.6$ \\
Sex $(\mathrm{M} / \mathrm{F})$ & $16 / 59$ & $23 / 45$ \\
PFTs \% pred & & \\
FEV1 & $90.1 \pm 6.8$ & $87.2 \pm 4.5$ \\
FVC & $90.7 \pm 1.0$ & $91.7 \pm 3.9$ \\
DLCO & $91.1 \pm 1.9$ & $80.4 \pm 2.1^{*}$ \\
Erythema nodosum (N/Y) & $\mathrm{NA}$ & $46 / 22$ \\
\hline Data arepresented &
\end{tabular}

Data are presented as mean \pm SD or as absolute numbers. * $\mathrm{p}<0.05$ as compared with controls. M/F: male/female; N/Y: No/Yes; NA: not applicable. 
and a previous diagnosis of autoimmune thyroid disease, and were under proper treatment when this study was performed. The diagnosis of thyroid disease preceded the diagnosis of sarcoidosis in all patients but one, in whom hypothyroidism due to Hashimoto's thyroiditis was diagnosed simultaneously.

We did not observe any case of clinical or subclinical AITD in the control group. The relationship between gender and thyroid disorders is well established, thus we stratified the two study groups according to gender.

\section{Female subjects (Table 2)}

Thyroid disease was present in 17 female sarcoidosis patients; 10 had autoimmune (Hashimoto's) thyroiditis with hypothyroidism, 2 had multinodular goiter, 3 had solitary thyroid nodule, 1 had Graves' disease and 1 had papillary follicular thyroid cancer. Thyroidectomy was performed in the patient with a history of thyroid cancer and the 3 patients with a history of multinodular goiter before this study was performed. We did not observe any new case of subclinical hypo- and/or hyperthyroidism in either of the groups.

Table 2. Laboratory measurements and ultrasonography results in female subjects

\begin{tabular}{lccc}
\hline Variable & $\begin{array}{c}\text { Controls } \\
(\mathbf{n = 5 9})\end{array}$ & $\begin{array}{c}\text { Sarcoidosis } \\
\text { patients } \\
(\mathbf{n = 4 5})\end{array}$ & p-value \\
\hline TSH $(\mu \mathrm{IU} / \mathrm{ml})$ & $1.67 \pm 0.68$ & $1.46 \pm 0.88$ & 0.17 \\
FT4 $(\mathrm{ng} / \mathrm{dl})$ & $1.18 \pm 0.11$ & $1.30 \pm 0.37$ & 0.2 \\
T3 $(\mathrm{ng} / \mathrm{ml})$ & $1.23 \pm 0.22$ & $1.28 \pm 0.19^{*}$ & 0.23 \\
TRAbs $(\mathrm{UI} / \mathrm{l})$ & $0.45 \pm 0.26$ & $1.98 \pm 2.59$ & 0.001 \\
TRAbs $(\%)$ & 0 & 40 & $<0.001$ \\
TPOAbs $(\mathrm{U} / \mathrm{ml})$ & $56.96 \pm 138.91$ & $100.34 \pm 198.91$ & 0.19 \\
TPOAbs $(\%)$ & 11.86 & 28.8 & 0.029 \\
TGAbs $(\mathrm{U} / \mathrm{ml})$ & $94.32 \pm 113.19$ & $118.54 \pm 350.82$ & 0.61 \\
TGAbs $(\%)$ & 5.08 & 6.6 & 0.73 \\
Thyroid volume $(\mathrm{ml})$ & $4.59 \pm 2.53$ & $3.91 \pm 2.37$ & 0.16 \\
Thyroid nodules & $30.5 \%$ & $39.02 \%$ & 0.48 \\
& $(18 / 59)$ & $(16 / 41)$ & \\
\hline
\end{tabular}

Values are presented as mean \pm SD or as percentages. NS: not significant. Normal values for TSH: $0.27-4.2 \mu \mathrm{IU} / \mathrm{ml}$, normal values for FT4: $0.7-2 \mathrm{ng} / \mathrm{dl}$, normal values for T3: 0.8-2ng/ml.

\section{Laboratory measurements}

There were no significant differences in TSH, FT4 and T3 levels in female patients compared to healthy volunteers (Table 2). Clinical AITD was present in 11 patients; 10 patients had Hashimoto's thyroiditis with hypothyroidism and 1 had Graves' disease. The TSH, FT4, AbTg, TPOAbs and TRAb values of the patients with Hashimoto's thyroiditis are $1.36 \pm 0.67,1.30 \pm 0.27$, $309.5 \pm 649.47,264.5 \pm 324.59$ and $1.91 \pm 1.33$, respectively. As for the patient with Graves', the TSH, FT4, AbTg, TPOAbs and TRAb values were 0.374, 1.17, 33.51, 69.66 and 6.17, respectively.

Female sarcoidosis patients exhibited higher incidence of serological autoimmunity (defined as the presence of increased titers of at least one thyroid antibody) vs controls $(68.8 \%$ vs $22.03 \%$, p $<0.001)$. TRAbs levels were significantly elevated in female sarcoidosis patients compared to healthy volunteers $(\mathrm{p}=0.001)$ (Table 2$)$ with $40 \%$ of the patients having positive TRAbs versus $0 \%$ of the controls $(\mathrm{p}<0.001)$. Interestingly, of the 18 female patients presenting positive TRabs titers, only 1 suffered from Graves' disease and 7 had Hashimoto's thyroiditis with hypothyroidism. The TSH, FT4, TGAbs, TPOAbs and TRAbs values of TRAb positive patients are $1.63 \pm 1.94,1.29 \pm 0.38,143.53 \pm 501,156.82 \pm 272.86$ and 3.52 \pm 3.12 , respectively. TPOAbs and TGAbs were higher in sarcoidosis patients; however, this did not reach statistical significance (Table 2). The frequency of positive TPOAbs was significantly increased in sarcoidosis vs healthy subjects $(28.8 \%$ vs $11.86 \%$, respectively, $p=0.029$ ). Of the 13 patients with elevated TPOAbs, 7 had Hashimoto's thyroiditis with hypothyroidism and 1 had Graves' disease. TGAbs were not significantly different when patients were compared with controls ( $6.6 \%$ vs $5.08 \%$, respectively, $\mathrm{p}>0.05)$.

Serum ACE levels were not different between patients with and without serological autoimmunity (46.94 \pm 17.74 vs $42.28 \pm 18.30$, respectively).

\section{Ultrasonography}

A higher percentage of female sarcoidosis patients presented with hypoechoic and dyshomogeneous echogenecity pattern of the thyroid gland when compared with controls $(75.6 \%$ vs $28.8 \%$, respectively, 
$\mathrm{p}<0.001)$. Sarcoidosis patients had more severe structural changes (in terms of dyshomogeneous echogenecity) of the thyroid gland vs healthy volunteers; however, this did not reach statistical significance (61.5\% vs $35.2 \%$, respectively, $p=0.092$ ). Sarcoidosis patients had increased frequency of thyroid nodules compared to controls; however, this did not reach statistical significance (Table 2). The vast majority $(94.4 \%)$ of female patients with increased TRAbs presented thyroid structural abnormalities (dyshomogeneous parenchyma and/or nodules). Thyroid volume did not differ between the two groups (Table 2). Overall, indices of thyroid autoimmune disease (increased titers of thyroid autoantibodies and/or ultrasonograhic hypoechoic pattern) were significantly more frequent in female sarcoidosis patients when compared to controls $(84.4 \%$ vs $32.2 \%, \mathrm{p}<0.001)$.

\section{Male subjects (Table 3)}

Thyroid disease was diagnosed in 3 male sarcoidosis patients before this study was performed; 2 patients presented multinodular goiter and 1 patient had a solitary thyroid nodule. One of the patients with multinodular goiter had been subjected to thyroidectomy.

\section{Laboratory measurements}

Basal TSH, FT4 and T3 levels were within normal

Table 3. Laboratory measurements and ultrasonography results in male subjects

\begin{tabular}{lccc}
\hline Variable & $\begin{array}{c}\text { Controls } \\
(\mathbf{n = 1 6})\end{array}$ & $\begin{array}{c}\text { Sarcoidosis } \\
\text { patients } \\
(\mathbf{n = 2 3})\end{array}$ & p-value \\
\hline TSH $(\mu \mathrm{IU} / \mathrm{ml})$ & $1.63 \pm 1.07$ & $1.68 \pm 1.99$ & 0.92 \\
FT4 $(\mathrm{ng} / \mathrm{dl})$ & $1.34 \pm 0.23$ & $1.31 \pm 0.25$ & 0.71 \\
T3 $(\mathrm{ng} / \mathrm{ml})$ & $1.13 \pm 0.17$ & $1.31 \pm 0.22$ & 0.67 \\
TRAbs $(\mathrm{UI} / \mathrm{l})$ & $0.45 \pm 0.32$ & $1.65 \pm 1.87$ & 0.006 \\
TRAbs $(\mathrm{UI} / \mathrm{l})(\%)$ & 0 & 43.4 & 0.002 \\
TPOAbs $(\mathrm{U} / \mathrm{ml})$ & $22.61 \pm 13.63$ & $56.79 \pm 105.49$ & 0.2 \\
TPOAbs $(\%)$ & 0 & 16.6 & 0.08 \\
TGAbs $(\mathrm{U} / \mathrm{ml})$ & $13.70 \pm 24.71$ & $41.22 \pm 69.69$ & 0.13 \\
TGAbs $(\%)$ & 0 & 4.1 & 0.39 \\
Thyroid volume $(\mathrm{ml})$ & $8.63 \pm 4.50$ & $6.62 \pm 2.71$ & 0.09 \\
Thyroid nodules & $62.5 \%$ & $45 . \%$ & 0.45 \\
\hline Values are presented & & & \\
\hline
\end{tabular}

Values are presented as mean \pm SD or as percentages. NS: not significant. Normal values for TSH: $0.27-4.2 \mu \mathrm{IU} / \mathrm{ml}$, normal values for FT4: $0.7-2 \mathrm{ng} / \mathrm{dl}$, normal values for T3: $0.8-2 \mathrm{ng} / \mathrm{ml}$. limits and did not differ significantly between male patients and controls (Table 3). Overall, male patients exhibited higher frequency of thyroid serological autoimmunity compared to controls $(47.8 \%$ vs $0 \%$, $\mathrm{p}=0.001)$. TRAbs levels were significantly higher in sarcoidosis patients compared to healthy volunteers $(p=0.006)$ (Table 3$)$. A significantly larger number of patients presented positive TRAbs compared to controls $(43.4 \%$ vs $0 \%, \mathrm{p}=0.002)$. The TSH, FT4, TGAbs, TPOAbs and TRAbs values of TRAb positive patients are $2.13 \pm 2.64,1.18 \pm 0.24,26.44 \pm 34.55$, $99.29 \pm 149.79$ and $3.22 \pm 1.9$, respectively. Of the male patients with positive TRAbs, $70 \%$ presented hypoechoic pattern in thyroid ultrasonography. TPOAbs and TGAbs were higher in the patient group, although this did not reach statistical significance (Table 3). Of the patients, $16.6 \%$ had positive TPOAbs and $4.1 \%$ had positive TGAbs but none of the controls had positive TPOAbs and/or TGAbs.

Serum ACE levels were not different between patients with and without serological autoimmunity (40.12 \pm 14.55 vs $41.54 \pm 18.35$, respectively, $p>0.05$ ).

\section{Ultrasonography}

Hypoechoic pattern of the thyroid gland was significantly more frequent in the patient group when compared with controls ( $40.9 \%$ vs $6.25 \%$, respectively, $\mathrm{p}=0.017$ ). Thyroid volume and the frequency of thyroid nodules did not differ between the two groups (Table 3). Overall, indices of thyroid autoimmune disease were significantly more frequent in sarcoidosis patients when compared to controls $(56.5 \%$ vs $6.2 \%$, $\mathrm{p}=0.001)$.

\section{FNA (female and male patients)}

Fine-needle aspiration was performed in 3 sarcoidosis patients with thyroid nodules with a diameter $>10 \mathrm{~mm}$. Cytological examination of the samples did not reveal the presence of non-caseating granulomas.

\section{Relationship between thyroid disease and various parameters in sarcoidosis patients}

The prevalence of thyroid antibodies in sarcoidosis patients with and without clinical autoimmune thyroid disease is presented in Table 4. All the patients with clinical AITD had evidence of serological autoimmunity (Table 4). When thyroid antibodies were examined 
Table 4. Association of clinical autoimmune thyroid disease with thyroid antibodies in sarcoidosis patients

\begin{tabular}{|c|c|c|c|}
\hline Variable & $\begin{array}{c}\text { No AITD } \\
(n=57)\end{array}$ & $\begin{array}{c}\text { With AITD } \\
(n=11)\end{array}$ & p value \\
\hline \multicolumn{4}{|l|}{ Serological autoimmunity* } \\
\hline No Abs & $45.61 \%$ & $0 \%$ & 0.004 \\
\hline At least $1 \mathrm{Ab}$ increased & $54.38 \%$ & $100 \%$ & \\
\hline \multicolumn{4}{|l|}{ TGAbs } \\
\hline Normal & $96.49 \%$ & $81.82 \%$ & 0.058 \\
\hline Increased & $3.51 \%$ & $18.18 \%$ & \\
\hline \multicolumn{4}{|l|}{ TPOAbs } \\
\hline Normal & $85.96 \%$ & $18.18 \%$ & $<0.001$ \\
\hline Increased & $14.04 \%$ & $81.82 \%$ & \\
\hline \multicolumn{4}{|l|}{ TRAbs } \\
\hline Normal & $57.89 \%$ & $27.27 \%$ & 0.062 \\
\hline Increased & $42.11 \%$ & $72.73 \%$ & \\
\hline \multicolumn{4}{|l|}{ Thyroid Hypoechoic } \\
\hline Pattern. & $50.88 \%$ & $18.18 \%$ & 0.046 \\
\hline No & $49.12 \%$ & $81.82 \%$ & \\
\hline Yes & & & \\
\hline \multicolumn{4}{|l|}{$\begin{array}{l}\text { Combination of serological } \\
\text { and/or ultarasonographic } \\
\text { autoimmunity }\end{array}$} \\
\hline No & $29.82 \%$ & 0 & 0.036 \\
\hline Yes & $70.18 \%$ & $100 \%$ & \\
\hline
\end{tabular}

Values are presented as percents *Serological autoimmunity was defined as increased titers of at least one thyroid antibody, $\cdot n=63$ due to thyroidectomy.

separately, TPOAbs were significantly associated with the presence of clinical autoimmune thyroid disease in sarcoidosis patients (Table 4). Autoimmune thyroid disease was significantly associated with the presence of hypoechoic and dyshomogeneous echogenecity of the thyroid gland $(p=0.046)$. Indices of serological and/or ultrasonographic autoimmunity were significantly higher in patients with clinical AITD (Table 4). We did not observe any significant association of erythema nodosum with indices of thyroid involvement or autoimmunity.

\section{DISCUSSION}

In our case control study, incorporating a very detailed examination of different thyroid parameters such as thyroid hormones and antibodies, thyroid ultrasonography and fine-needle aspiration of the nodules, we have demonstrated a significant associa- tion between sarcoidosis and autoimmune thyroid disease. Sarcoidosis patients in our study exhibited higher incidence of serological thyroid autoimmunity, with increased frequency of positive TRAbs and TPOAbs. Additionally, sarcoidosis was associated with increased frequency of structural changes of the thyroid gland suggestive of thyroid autoimmunity. This association of sarcoidosis with autoimmune thyroid diseases may reflect a common pathogenetic background between the two entities.

The association of sarcoidosis with thyroid disorders has received significant attention in recent years. Granuloma infiltration of the thyroid gland is considered to be rare, with a prevalence of $4 \%$ in large series of autopsied patients with systemic sarcoidosis. ${ }^{17}$ No such evidence derived from our study; however, we only performed FNAs to nodules greater than $10 \mathrm{~mm}$, and thus infiltration of some of the glands cannot be excluded. Previous studies have also reported a high frequency of autoimmune thyroid disease in patients with sarcoidosis, ranging from $2.9 \%$ to $10.2 \% .^{18,19}$ In the literature, clinical hypothyroidism was documented in 5.3\% and Graves' disease in $4 \%$ of the female sarcoidosis patients. ${ }^{8}$ In our cohort, the overall frequency of thyroid disorders was $29.4 \%$, with $16.1 \%$ of our patients suffering from clinical AITD. Our findings support the concept that sarcoidosis is associated with a higher frequency of thyroid dysfunction and autoimmune thyroid disease. However, we observed a higher frequency of AITD in our population of sarcoidosis patients compared with previous reports in the literature. This difference might be due to different iodine intake in the different populations studied and/or discrepancies in the methods used to assess the thyroid status. As thyroid disease was diagnosed in all but one of the cases before the diagnosis of sarcoidosis, patients with previously abnormal thyroid function test were on treatment and were euthyroid at the time of the examination.

Studies in the literature examining the frequency of thyroid antibodies in sarcoidosis are conflicting and rather scarce. Studies in small cohorts have failed to document the presence of increased levels of TPOAbs in sarcoidosis patients. ${ }^{11}$ However, larger studies have reported increased levels of TPOAbs in sarcoidosis 
patients. ${ }^{8,10}$ In agreement with the latter, we also found significantly increased TPOAbs in female sarcoidosis patients compared to controls, and TPOAbs were significantly associated with clinical AITD.

The data regarding the presence of TRAbs in sarcoidosis are somewhat scarce and mainly consist of case reports. ${ }^{20}$ Data derived from larger studies have not shown positive TRAbs in sarcoidosis. ${ }^{12}$ However, our findings show a significantly higher frequency of TRAbs in sarcoidosis patients compared to gendermatched controls. Moreover, in our study the presence of TRAbs was almost unequivocally associated with ultrasonographic signs of thyroid autoimmunity (dyshomogeneous thyroid parenchyma). Differences in the methods used for antibody estimation and age differences might be responsible for the conflicting results. Furthermore, none of our patients had received any steroid or other immunosuppressive treatment for a year before the study, though in other studies patients under such treatment were included. Studies have shown that TRAbs are present in 5-10\% of patients with hypothyroidism associated with Hashimoto's thyroiditis and $80-95 \%$ of patients with Graves' disease..$^{21}$ The increased frequency of TRAbs in Hashimoto's thyroiditis in our sarcoidosis patients may represent a more widely distributed characteristic of abnormal autoimmune function associated with the disease, since no positive TRAbs were observed in the control group. In autoimmune thyroid disease, TRAbs are considered an example of a primary autoantibody that causes the disease rather than being secondary to it. ${ }^{22}$ In contrast to TPOAbs and TGAbs, TRAbs are never found in the apparently euthyroid "normal" population. Although the clinical significance of the elevated TRAbs in the sarcoid population has yet to be determined, our study demonstrated that they are associated with thyroid structural changes. Taking the above into account, one may speculate that the elevation of TRAbs could possibly be an indicator of subclinical or a high-risk predicting factor for thyroid autoimmune disorders in these patients. Therefore, sarcoidosis patients with elevated TRAbs may deserve a close thyroid function follow-up. Clearly, additional studies are warranted in order to confirm the aforementioned hypothesis.

Apart from thyroid antibodies, studies have shown a high incidence of elevated titers of antinuclear anti- bodies and rheumatoid factor ${ }^{7}$ as well as antibodies to double-stranded DNA ${ }^{5}$ in sarcoidosis. Sarcoid granuloma formation is the result of a complex interplay of numerous factors. ${ }^{6}$ According to current concepts, the immunologic diversions involved in sarcoidosis pathobiology include persistent antigenemia, a hypereactive CD4-helper cell recognition, an effusive humoral B-cell immune reaction and the existence of circulating immune-complexes. ${ }^{6}$ Sarcoidosis is associated with an exaggerated $\mathrm{T}$ helper-cell immune response and increased amounts of circulating immunoglobulins, among which are antibodies for self-antigens, therefore autoantibody production is considered to be enhanced. These immune dysregulations associated with the disease under the combined action of genetic and environmental conditions may induce the appearance of multiple immune mediated disorders, leading to different clinical disorders, including AITD. Although the exact mechanism of the production of thyroid antibodies in sarcoidosis remains to be elucidated, our study supports the hypothesis that the association of sarcoidosis with thyroid disorders has an immunologic nature.

Thyroid antibodies may be present in the general population without evidence of thyroid dysfunction. In our cohort we found higher serological thyroid autoimmunity in sarcoidosis, and the presence of positive antibodies was significantly associated with the presence of clinical AITD and ultrasonographic findings of thyroid autoimmunity. This may potentially suggest that the presence of thyroid antibodies may be clinically significant in sarcoidosis because they may indicate a significantly higher risk of autoimmune thyroid diseases in this population. Furthermore, serological autoimmunity in sarcoidosis may be associated with a more severe and aggressive thyroiditis. However, this constitutes merely a speculation and further studies addressing this hypothesis are certainly warranted.

The association of sarcoidosis with thyroid cancer was not confirmed in our cohort. We reported only one patient presenting papillary follicular thyroid cancer. To our knowledge, the association of sarcoidosis with thyroid carcinoma has been shown only in case reports and, clearly, larger studies are needed in order to examine the link between the two disease entities. Additionally, we did not document increased 
prevalence of thyroid nodules in sarcoidosis. Our findings are in accordance with previous studies. ${ }^{8}$

In conclusion, we demonstrated a rather high prevalence of thyroid diseases in sarcoidosis patients. Additionally, we observed that indices of serological and ultrasonographic thyroid autoimmunity were significantly increased in sarcoidosis, suggesting that the association of the disease with thyroid disorders may have an immunological background. The clinical significance of thyroid autoimmunity in sarcoidosis has not as yet been clarified, and we believe that further investigations are required in order to investigate the mechanisms and the pathophysiological complications of our observations.

\section{REFERENCES}

1. Statement on sarcoidosis. Joint Statement of the American Thoracic Society (ATS), the European Respiratory Society (ERS) and the World Association of Sarcoidosis and Other Granulomatous Disorders (WASOG) adopted by the ATS Board of Directors and by the ERS Executive Committee, February 1999. Am J Respir Crit Care Med 160: 736-755.

2. Mathur A, Kremer JM, 1993 Immunopathology, musculoskeletal features, and treatment of sarcoidosis. Curr Opin Rheumatol 5: 90-94.

3. Hunninghake GW, Crystal RG, 1981 Mechanisms of hypergammaglobulinemia in pulmonary sarcoidosis. Site of increased antibody production and role of $\mathrm{T}$ lymphocytes. J Clin Invest 67: 86-92.

4. Porter N, Beynon HL, Randeva HS, 2003 Endocrine and reproductive manifestations of sarcoidosis. QJM 96: 553-561.

5. Weinberg I, Vasiliev L, Gotsman I, 2000 Anti-dsDNA antibodies in sarcoidosis. Semin Arthritis Rheum 29: 328-331.

6. Sharma OP, 2002 Sarcoidosis and other autoimmune disorders. Curr Opin Pulm Med 8: 452-456.

7. Giotaki HA, 1989 Biochemical and immunological parameters of sarcoid patients in west Greece. Sarcoidosis 6: $135-137$.

8. Antonelli A, Fazzi P, Fallahi P, Ferrari SM, Ferrannini E, 2006 Prevalence of hypothyroidism and Graves disease in sarcoidosis. Chest 130: 526-532.
9. Barnadas MA, Rodriguez-Arias JM, Alomar A, 2000 Subcutaneous sarcoidosis associated with vitiligo, pernicious anaemia and autoimmune thyroiditis. Clin Exp Dermatol 25: 55-56.

10. Nakamura H, Genma R, Mikami T, et al, 1997 High incidence of positive autoantibodies against thyroid peroxidase and thyroglobulin in patients with sarcoidosis. Clin Endocrinol (Oxf) 46: 467-472.

11. Ilias I, Panoutsopoulos G, Batsakis C, Nikolakakou D, Filippou N, Christakopoulou I, 1998 Thyroid function and autoimmunity in sarcoidosis: a case-control study. Croat Med J 39: 404-406.

12. Antonelli A, Fazzi P, Fallahi P, et al, 2009 Thyroid uptake of $67 \mathrm{Ga}$-citrate is associated with thyroid autoimmunity and hypothyroidism in patients with sarcoidosis. Eur J Nucl Med Mol Imaging 36: 137-143.

13. Fountoulakis S, Philippou G, Tsatsoulis A, 2007 The role of iodine in the evolution of thyroid disease in Greece: from endemic goiter to thyroid autoimmunity. Hormones (Athens) 6: 25-35.

14. Koutras DA, Alevizaki M, Tsatsoulis A, Vagenakis AG, 2003 Greece is iodine sufficient. Lancet 362: 405-6.

15. Shabana W, Peeters E, De Maeseneer M, 2006 Measuring thyroid gland volume: should we change the correction factor? AJR Am J Roentgenol 186: 234-236.

16. Antonelli A, Fallahi P, Nesti C, et al, 2001 Anti-CD38 autoimmunity in patients with chronic autoimmune thyroiditis or Graves' disease. Clin Exp Immunol 126: 426-431.

17. Attali JR, Valensi P, Valeyre D, Sandre-Banon D, Sebaoun J, Battesti JP, 1994 Thyroid stimulating antibodies in sarcoidosis. Pathol Biol (Paris) 42: 581-586.

18. Isern V, Lora-Tamayo J, Capdevila O, Villabona C, Mana J, 2007 Sarcoidosis and autoimmune thyroid disease. A case series of ten patients. Sarcoidosis Vasc Diffuse Lung Dis 24: 148-152.

19. Papadopoulos KI, Hornblad Y, Liljebladh H, Hallengren B, 1996 High frequency of endocrine autoimmunity in patients with sarcoidosis. Eur J Endocrinol 134: 331336.

20. Yarman S, Kahraman H, Tanakol R, Kapran Y, 2003 Concomitant association of thyroid sarcoidosis and Graves' disease. Horm Res 59: 43-46.

21. Zophel K, Roggenbuck D, Schott M, 2010 Clinical review about TRAb assay's history. Autoimmun Rev 9: 695-700.

22. Sinclair D, 2008 Analytical aspects of thyroid antibodies estimation. Autoimmunity 41: 46-54. 